\title{
Quimioprofilaxis en niños y adultos sometidos a trasplante de órganos sólidos y precursores hematopoyéticos. Recomendaciones del Comité de Infecciones en Inmunocomprometidos Sociedad Chilena de Infectología. 2011
}

\author{
Chemoprophylaxis in children and adults undergoing solid organ and \\ hematopoietic stem cells transplantation. \\ Recommendations of The Committee of Infections in Immunocompromised Patients \\ of the Chilean Society of Infectious Diseases. 2011
}

Alba G., Andrea

Unidad de Segunda Infancia

Hospital Dr. Luis Calvo Mackenna, Santiago.

Álvarez P., Ana M.

Servicio de Pediatría

Hospital San Juan de Dios,

Santiago.

Avilés L., Carmen L.

Departamento de Pediatría

Hospital San Borja-Arriarán

Facultad de Medicina,

Universidad de Chile, Santiago.

Benitez G., Rosana

Servicio de Medicina

Hospital DIPRECA,

Santiago.

Bidart H., Teresa

Unidad de Infectología

Clínica Santa María,

Santiago.

Catalán M., Paula

Unidad de Trasplante de Precursores

Hematopoyéticos

Hospital Dr. Luis Calvo Mackenna,

Santiago.

Coordinadora: M. Pilar Gambra A.

Integrantes del consenso (en orden alfabético)

Ferrés G., Marcela

Laboratorio de Infectología y

Virología Molecular

Escuela de Medicina, Pontificia

Universidad Católica de Chile,

Santiago.

Gambra A., M. Pilar

Unidad de Infectología,

Hospital Barros Luco Trudeau,

Santiago.

Facultad de Medicina, Universidad

de Chile, Santiago.

Lafourcade R., Mónica

Laboratorio de Microbiología

Clínica Santa María,

Santiago.

Nervi N., Bruno

Departamento de Oncología

Escuela de Medicina

Pontificia Universidad Católica de Chile,

Santiago.

\section{Noemi H., Isabel}

Departamento de Pediatría

Hospital Dr. Luis Calvo Mackenna,

Santiago.

Facultad de Medicina, Universidad

de Chile.
Payá G., Ernesto

Servicio de Pediatría

Hospital de Carabineros

Universidad Mayor, Santiago.

Rabagliati B., Ricardo

Departamento de Enfermedades

Infecciosas, Escuela de Medicina

Pontificia Universidad Católica de Chile,

Santiago.

Ramírez V., Pablo

Departamento de Oncología

Escuela de Medicina

Pontificia Universidad Católica de Chile,

Santiago.

Santolaya de P., M. Elena

Unidad de Infectología

Hospital Dr. Luis Calvo Mackenna,

Santiago

Facultad de Medicina, Universidad de Chile.

Tassara O., Renzo

Departamento de Pediatría

Hospital San Juan de Dios, Santiago

Facultad de Medicina, Universidad de Chile.

Zubieta A., Marcela

Servicio de Pediatría

Hospital Exequiel González Cortés,

Santiago. 


\section{Introducción}

El envejecimiento de la población y el avance de la medicina han permitido que el trasplante de órganos sólidos y precursores hematopoyéticos sean parte de la atención de un número creciente de personas. El trasplante predispone a infecciones dependiendo del órgano trasplantado, la inmunosupresión requerida, de la situación particular del binomio donante-receptor así como de la realidad epidemiológica del país. Las infecciones en el paciente trasplantado pueden comprometer tanto la viabilidad del trasplante como la vida del paciente. La complejidad de las medidas de prevención sugeridas o establecidas en la literatura científica generan confusión en los profesionales de atención de salud, por lo que surgió la necesidad de realizar recomendaciones que permitan estandarizar las opciones de prevención farmacológicas disponibles en la actualidad y adaptarlas a la realidad nacional. Estas recomendaciones representan una guía basada en la evidencia y revisan la epidemiología, factores de riesgo y medidas de prevención farmacológicas de enfermedades fúngicas, virales, parasitarias y micobacterianas más importantes en pacientes adultos y pediátricos sometidos a trasplantes de órganos sólidos y precursores hematopoyéticos. El siguiente documento pretende asistir al médico en la toma de decisiones prácticas, válidas, reproducibles y clínicamente flexibles en relación a quimioprofilaxis (QP) pre y post-trasplante.

Metodología. Se constituyó un panel de médicos expertos en microbiología clínica, infectólogos de adultos y niños, especialistas en trasplante de órganos sólidos (TOS) y trasplante de precursores hematopoyéticos (TPH).

Entre marzo y noviembre de 2010 este comité analizó la literatura científica publicada sobre QP en pacientes receptores de TOS y TPH, en idiomas inglés y español durante los últimos 10 años. Se realizó una búsqueda sistemática con los términos MESH: Transplant OR trasplant AND prophylaxis OR chemoprophylaxis, en los siguientes temas:

- Hongos: Candida, hongos filamentosos, Pneumocystis jiroveci.

- Virus: citomegalovirus, virus de Epstein-Barr, adenovirus, virus influenza, virus respiratorio sincicial.

- Parásitos: Toxoplasma gondii, Trypanosoma cruzi (enfermedad de Chagas).

- Micobacterias: Mycobacterium tuberculosis.

En cada área temática se intentó responder las interrogantes relacionadas a: epidemiología, factores de riesgo, eficacia de la quimioprofilaxis, fármacos empleados y dosis, duración de la QP y seguimiento.

Se clasificó las recomendaciones según la calidad y fuerza de la evidencia (Tabla anexa).

María Pilar Gambra Acle Coordinadora pilargambra@yahoo.es

Clasificación de grados de recomendación y calidad de la evidencia utilizados*

Definición de grados de recomendación

\begin{tabular}{|c|c|}
\hline A & Buena evidencia para recomendar su uso \\
\hline B & Moderada evidencia para recomendar su uso \\
\hline C & Pobre evidencia para recomendar su uso \\
\hline D & Moderada evidencia para desaconsejar su uso \\
\hline $\mathrm{E}$ & Buena evidencia para desaconsejar su uso \\
\hline \multicolumn{2}{|c|}{ Calidad de la evidencia } \\
\hline I & Uno o más trabajos prospectivos, randomizados y controlados \\
\hline II & $\begin{array}{l}\text { - Uno o más estudios clínicos, no randomizados } \\
\text { - Uno o más estudios de cohorte o caso-control (idealmente multicéntrico) } \\
\text { - Múltiples series de casos } \\
\text { - Experimentos no controlados significativos }\end{array}$ \\
\hline III & Opiniones de expertos, basadas en experiencias clínicas, estudios descriptivos o reportes de comités. \\
\hline
\end{tabular}




\section{Introduction}

Aging of the population and medical advances have made solid organ and hematopoietic stem cells transplants part of the care of a growing number of people. Transplant predisposes to infection depending on its type, immunosuppression required, donor-recipient conditions, as well as the epidemiological situation in the country. Infections in these patients can compromise both viability of the transplant itself as the patient's life. The complexity of prevention can generate confusion in health care personnel. For this reason, it is necessary to make recommendations standardizing currently available pharmacological prevention options and adapt them to our national reality. These evidence based guidelines review the epidemiology, risk factors and pharmacological prevention measures for the most important fungal, viral, parasitic and mycobacterial diseases in solid organ and hematopoietic stem cells transplants, both in adults and children.

Methods. An Expert Committee of microbiologists, infectious diseases specialists, solid organs and hematopoietic stem cells transplantation specialists was constituted.

From March to November 2010 this Committee reviewed and analyzed scientific publications available about chemoprophylaxis for receptors of solid organ and hematopoietic stem cells transplants. Established limits for this review were articles published during last ten years in English or Spanish languages. Utilized MESH terms were: Transplant OR trasplant AND prophylaxis OR chemoprophylaxis, in the following topics:

- Fungus: Candida, molds, Pneumocystis jiroveci.

- Virus: citomegalovirus, Epstein-Barr virus, adenovirus, influenza virus, respiratory syncytial virus.

- Parasites: Toxoplasma gondii, Trypanosoma cruzi (Chagas disease).

- Mycobacteria: Mycobacterium tuberculosis.

For each microorganism the following aspects were searched in order to answer queries related to: epidemiology, risk factors, chemoprophylaxis efficacy, drugs and schedules recommended and follow up.

Each recommendation was classified according to the scientific quality of the evidence and the emphasis done to this recommendation.

María Pilar Gambra Acle Coordinadora pilargambra@yahoo.es

\begin{tabular}{|ll|ll}
\multicolumn{2}{c}{ Abreviaturas utilizadas en el documento } \\
\hline ADN & Ácido desoxiribonucleico & $\mathrm{mg}$ & miligramo \\
ADV & Adenovirus & $\mathrm{PI}$ & Parainfluenza \\
CMV & Citomegalovirus & $\mathrm{R}(-)$ & Receptor negativo \\
dl & Decilitro & $\mathrm{R}(+)$ & Receptor positivo \\
$\mathrm{D}(-)$ & Donante negativo & $\mathrm{RPC}$ & Reacción de polimerasa en cadena \\
$\mathrm{D}(+)$ & Donante positivo & $\mathrm{SLP}$ & Síndrome linfoproliferativo \\
EFI & Enfermedad fúngica invasora & TL4 & Linfocitos T CD4 \\
EICH & Enfermedad de injerto contra hospedero & TOS & Trasplante de órganos sólidos \\
FLU & Influenza & TPH & Trasplante de precursores hematopoyéticos \\
h & hora $(s)$ & UCl & Unidad de cuidados intensivos \\
iv & intravenosa & vo & vía oral \\
LLA & Leucemia linfoblástica aguda & VRS & Virus respiratorio sincicial \\
\hline
\end{tabular}

